\title{
Presencia y ubicación de los portales de transparencia municipales españoles
}

\section{Presence and location of Spanish municipal transparency portals}

\author{
Pilar Beltrán-Orenes; David Rodríguez-Mateos
}

Cómo citar este artículo:

Beltrán-Orenes, Pilar; Rodríguez-Mateos, David (2020). “Presencia y ubicación de los portales de transparencia municipales españoles". Profesional de la información, v. 29, n. 6, e290635.

https://doi.org/10.3145/epi.2020.nov.35

Artículo recibido el 11-06-2020

Aceptación definitiva: 28-07-2020

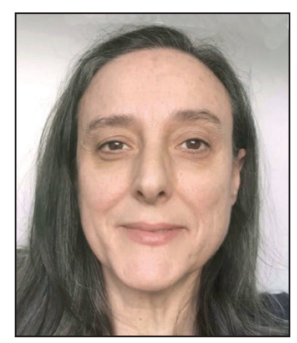

\author{
Pilar Beltrán-Orenes $\bowtie$ \\ https://orcid.org/0000-0002-1408-6365 \\ Universidad Rey Juan Carlos \\ Facultad de Ciencias de la Comunicación \\ Camino del Molino, 5 \\ 28942 Fuenlabrada (Madrid), España \\ pilar.beltran@urjc.es
}

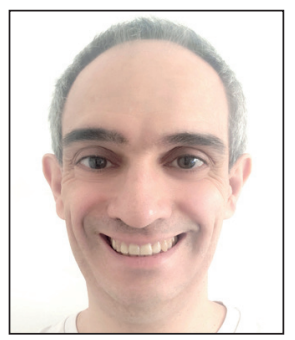

David Rodríguez-Mateos
https://orcid.org/0000-0003-1555-5685
Universidad Carlos III de Madrid
Facultad de Humanidades, Comunicación
y Documentación
Departamento de Comunicación
28903 Getafe (Madrid), España
pirio@bib.uc3m.es

\section{Resumen}

Desde 2015 los ayuntamientos españoles deben cumplir con la Ley de transparencia, que incluye el deber de publicar información sobre su actividad en una web, sede electrónica o portal de transparencia. Para ello, cuentan en muchos casos con la ayuda de diputaciones provinciales, comunidades autónomas o el Estado. Los estudios previos sobre el cumplimiento de esta norma han dejado aparte, en buena parte de los casos, a los ayuntamientos menores de 10.000 habitantes, es decir, a más del $90 \%$. Este trabajo ofrece un análisis introductorio sobre la presencia de información de transparencia en webs y portales de transparencia ofrecida por ayuntamientos españoles de todos los rangos de población. Se ha estudiado una muestra de municipios, divididos en catorce rangos, que abarca entre un 20 y un $30 \%$ de municipios, extraídos proporcional y aleatoriamente para cada rango y cada provincia. El análisis incluye variables como la existencia de sitios web municipales y de portales de transparencia específicos, la conexión y publicidad entre ambos, y la presencia mínima de información dentro de los portales de transparencia. Los resultados muestran que, a pesar del apoyo de instituciones de ámbito mayor, uno de cada seis ayuntamientos no tiene web, y la mitad de los municipios no ofrece información real sobre transparencia. Esto es debido a tres causas: sus portales de transparencia están vacíos, no hacen publicidad activa de su portal en la web o, en menor medida (uno de cada diez) no tienen portal de transparencia. Este resultado ocurre en los municipios de menos de 20.000 habitantes. En cuanto a los municipios que sí ofrecen información sobre transparencia, emplean sobre todo la aplicación facilitada por el Estado que está asociada a una sede electrónica. La actividad de otras administraciones (autonómicas o provinciales) para fomentar esta transparencia municipal también aparece, aunque sólo de forma masiva en algunas provincias.

\section{Palabras clave}

Transparencia local; Ayuntamientos; Portales de transparencia; Rangos de población; Sitios web; Webs; Información pública; Sede electrónica; Publicidad activa; Ley de transparencia; Muestreo; Entidades locales.

\begin{abstract}
Since 2015, Spanish local councils have had to comply with the Transparency Act, which includes the duty to publish information on their activity on a website, electronic headquarters, or transparency portal. To do so, they often have the help of provincial councils, autonomous communities, or the State. Previous studies on compliance with this rule ignored town councils with fewer than 10,000 inhabitants, i.e., more than $90 \%$ of them. This paper presents an introductory analysis on the presence of transparency information on websites and transparency portals offered by municipalities of all populations. A sample of municipalities, divided into 14 population ranges, was studied, covering $20-30 \%$ of municipalities, extracted proportionally and randomly for each range and from each province. The analysis includes variables
\end{abstract}


such as the existence of a municipal website and specific transparency portal, the connection and publicity between the two, and the minimum presence of information within such portals. Despite support from larger institutions, one out of six municipalities did not have a website, and half of the municipalities did not offer real information on transparency. The three main causes identified (in order) are that their transparency portals are empty, that they do not actively advertise their portal on the website, or to a lesser extent (one in ten) that they do not have a transparency portal. This result occurs mostly for municipalities with fewer than 20,000 inhabitants. The municipalities that do offer transparency information mainly use tools provided by the State, which are associated with an electronic site. Efforts by other (autonomous or provincial) institutions to promote transparency are also detected, although only massively in some provinces.

\section{Keywords}

Local transparency; City councils; Transparency portals; Population ranges; Websites; Public information; Electronic office "Sede electrónica"; Active advertising; Transparency law; Sampling; Local entities.

\section{La transparencia local en España}

Las administraciones locales, como todas las administraciones públicas en España, están obligadas por ley a publicar

"de forma periódica y actualizada la información cuyo conocimiento sea relevante para garantizar la transparencia de su actividad relacionada con el funcionamiento y control de la actuación pública",

según el artículo 5.1 de la Ley 19/2013, de 9 de diciembre, de transparencia, acceso a la información pública y buen gobierno (LTBG) (España, 2013). En su artículo 5.4, se añade que

"la información sujeta a las obligaciones de transparencia será publicada en las correspondientes sedes electrónicas o web y de una manera clara, estructurada y entendible para los interesados y, preferiblemente, en formatos reutilizables".

Además, se estableció una demora de dos años (Guichot, 2015) para estas administraciones. Este plazo fue aprovechado para definir pautas y tecnologías destinadas a la aplicación de la ley. Por ejemplo, la "Ordenanza tipo de transparencia, acceso a la información y reutilización" (FEMP, 2014) señalaba:

“1. La información se publicará en la página web o sede electrónica de las entidades incluidas en su ámbito de aplicación, o, en su caso, en un portal específico de transparencia.

$[\ldots]$

2. La entidad local podrá adoptar otras medidas complementarias y de colaboración con el resto de administraciones públicas para el cumplimiento de sus obligaciones de publicidad activa, incluyendo la utilización de portales de transparencia y de datos de otras entidades" (artículo 13.1 y 13.2).

\subsection{Algunas dificultades: dispersión de la población en municipios pequeños}

El flanco más débil en la aplicación de la transparencia municipal lo constituyen los ayuntamientos más pequeños:

"La multiplicidad de las entidades que integran la Administración Local española, la gran mayoría de ellas con población inferior a 20.000 habitantes, imposibilitan, o hacen muy difícil que muchas de ellas, sobre todo las de menor población, puedan desarrollar adecuadamente todas las obligaciones que establecen las diferentes leyes. Por ello la Ley 7/85 Reguladora de las Bases de Régimen Local atribuye a las Diputaciones Provinciales o entidades equivalentes una serie de competencias de coordinación y asistencia a los municipios, sobre todo los de población inferior a 20.000 habitantes. Por la naturaleza de las obligaciones que Ley 19/2013, de 9 de diciembre, impone a las entidades que integran la Administración Local, se hace necesario que sean las Diputaciones Provinciales o entidades equivalentes las que colaboren con esas entidades para poder cumplir con lo establecido en la Ley de transparencia y buen gobierno" (Minhap-Femp, 2015)

En particular, deben reseñarse algunos datos al respecto. En primer lugar, la gran mayoría de los municipios españoles son, no ya inferiores a 20.000 habitantes, sino a 10.000 (tabla 1).

Pese a la alta concentración de la población española en municipios mayores, algo más del 20\% de los habitantes residen en estos municipios (tabla 2).

Tabla 1. Número y porcentaje de municipios de $\leq 10.000$ habitantes en España. Fuente: INE, 2020.

https://tinyurl.com/yxyg $477 q$

\begin{tabular}{|c|c|}
\hline $\mathbf{N}$ total de municipios & $\mathbf{5} \mathbf{1 0 . 0 0 0}$ habitantes \\
\hline 8.131 & 7.378 \\
\hline $100 \%$ & $90,74 \%$ \\
\hline
\end{tabular}

Tabla 2. Número y porcentaje de la población de $>$ y $\leq 10.000$ habitantes en España. Fuente: INE, 2020. https://tinyurl.com/yxyg $477 q$

\begin{tabular}{|c|c|c|}
\hline Población total & $\mathbf{> 1 0 . 0 0 0}$ habitantes & $\mathbf{\leq 1 0 . 0 0 0}$ habitantes \\
\hline 47.026 .208 & 37.460 .754 & 9.565 .454 \\
\hline $100 \%$ & $79,66 \%$ & $20,34 \%$ \\
\hline
\end{tabular}


Tabla 3. Número y porcentaje de municipios de $\leq 20.000$ habitantes en España. Fuente: INE, 2020.

https://tinyurl.com/yxyg $477 q$

\begin{tabular}{|c|c|}
\hline $\mathbf{N}$ total de municipios & $\mathbf{5} \mathbf{2 0 . 0 0 0}$ habitantes \\
\hline 8.131 & 7.718 \\
\hline $100 \%$ & $95 \%$ \\
\hline
\end{tabular}

Tabla 4. Número y porcentaje de la población de $>$ y $\leq 10.000$ habitantes en España. Fuente: INE, 2020. https://tinyurl.com/yxyg477q

\begin{tabular}{|c|c|c|}
\hline Población total & $\mathbf{2} \mathbf{2 0 . 0 0 0}$ habitantes & $\mathbf{5} \mathbf{2 0 . 0 0 0}$ habitantes \\
\hline 47.026 .208 & 32.672 .973 & 14.353 .235 \\
\hline $100 \%$ & $\mathbf{6 9 , 5} \%$ & $\mathbf{3 0 , 5} \%$ \\
\hline
\end{tabular}

Además, el 95\% de los municipios españoles está por debajo de los 20.001 habitantes (tabla 3).

Y en ellos residen 3 de cada 10 españoles (tabla 4).

\subsection{Iniciativas estatales para fomentar la transparencia municipal}

La Administración General del Estado (AGE) ha tratado de promover la transparencia municipal mediante algunas iniciativas. Por un lado, el 28 de abril de 2015 se firmó el “Acuerdo marco de colaboración entre la Administración General del Estado (Ministerio de Hacienda y Administraciones Públicas [actual Ministerio de Política Territorial y Función Pública]) y la Federación Española de Municipios y Provincias (FEMP) para promover y facilitar el desarrollo de la Ley 19/2013, de 9 de diciembre, de transparencia, acceso a la información pública y buen gobierno en las entidades locales" (Minhap-Femp, 2015), con especial mención a los municipios de menos de 20.000 habitantes, tal como se recoge en la cita del apartado anterior.

Se inicia, así, una experiencia piloto de soporte estatal de portales de transparencia a las entidades locales (EELL) (gob. es) con

"una estructura análoga a la del Portal de Transparencia de la Administración General del Estado [...] Dicho portal (en adelante Portal de la Transparencia Local) conforme a un modelo de servicio común dispondrá de los siguientes servicios generales:

1. Gestión de solicitudes de acceso.

2. Publicidad activa centralizada.

3. Publicidad activa descentralizada (TR-Utils).

4. Buscador e indexador de la información.

5. Gestor de contenidos del portal" (Minhap-Femp, 2015: Cláusula segunda-obligaciones que asume el Minhap).

Aunque la iniciativa sigue activa, en la actualidad, apenas 518 EELL lo siguen usando. Véase la web "Portales de Transparencia Local:

https://directorio.transparencialocal.gob.es/es_ES

y de ellas, 148 se encuentran en mantenimiento.

Por otra parte, en 2014 se creó la sede electrónica de la AGE, que incluye un portal de transparencia entre sus contenidos y funcionalidades, tal como recoge el art. 3.1.d de la Orden HAP/1949/2014, de 13 de octubre, por la que se regula el Punto de Acceso General de la Administración General del Estado [PAe] y se crea su sede electrónica (España, 2014) y en el que se explicita que

"existe una gran dispersión de la información de las Administraciones en distintos portales y webs, que provoca dificultades en el acceso de los ciudadanos a los procedimientos y servicios administrativos, informaciones duplicadas y falta de una coordinación adecuada en todas estas materias" ${ }^{\prime 2}$.

Aunque el portal de transparencia local proporcionado por la AGE a las EELL era inicialmente el gob.es (Minhap-Femp, 2015), los distintos convenios de colaboración firmados entre las Comunidades Autónomas (CCAA) y la AGE para la prestación mutua de soporte en administración electrónica $(P A e, 2019)$ han tenido como consecuencia el uso del portal de transparencia de la sede electrónica, en detrimento del gob.es, por parte de muchas EELL.

No obstante, en este segundo caso, no se ha podido localizar información oficial sobre cuántos municipios se han sumado a esta iniciativa y están realmente empleando este modelo de sede electrónica, ni acerca de si están empleando el portal de transparencia que va incluido en ella.

\subsection{Dificultades para localizar la información sobre transparencia municipal en España}

Además de los sistemas de origen estatal, diversas administraciones autonómicas y municipales han tratado de dar soporte a sus municipios mediante herramientas propias, para fomentar su transparencia. Cada iniciativa, además, propone su sistema de organización que, a la postre, determina posibilidad de la evaluación posterior de su cumplimiento. Como en el caso de las opciones de la AGE, cada municipio tiene plena autonomía para decidir cómo y dónde publica su información para cumplir con los requisitos de transparencia.

Así pues, para abordar la evaluación de la transparencia municipal, antes se han de tener en cuenta algunos aspectos, tanto de ubicación como tecnológicos. En primer lugar, si la información está en un recurso tecnológico que controle el propio ayuntamiento, como una sede web propia; o bien, si está alojada en un recurso de otra institución superior (ya 
sea una diputación provincial, una comunidad autónoma o la AGE), siendo el propio municipio el que pone la información en ambos casos.

En segundo lugar, si esa información se compila en un portal de transparencia, con unas categorías y organización preestablecida, o bien, en la web del municipio, y dentro de ella, si está agrupada en alguna sección, o está dispersa dentro de esa web, en ambos casos proporcionada por el municipio. Y una tercera opción, especialmente para municipios pequeños, si la información de los municipios está compilada por otra institución (como una diputación provincial o comunidad autónoma), y no por el propio municipio, sin que, en muchos casos, haya conexión entre la web municipal y su información sobre transparencia.

Todos estos factores suponen que resulte, en muchos casos, verdaderamente ardua la localización, no ya de los contenidos, sino de la propia ubicación de la información de transparencia, lo cual no resulta transparente. A ello se suma un argumento más: existen ayuntamientos que tienen información sobre transparencia en más de una ubicación al mismo tiempo: por ejemplo, en dos portales de transparencia, sin que se indique en ningún caso esta doble existencia.

Hemos de añadir que no en todos los casos es posible acceder a directorios de acceso a estos recursos, agrupados de forma territorial, o bien, estos directorios no están actualizados, o no incluyen acceso a todos los posibles recursos sobre transparencia de un municipio. De hecho, solo Cataluña dispone de un directorio específico que contiene todos los portales de transparencia de sus municipios, así como el enlace a ellos, independientemente de la ubicación y el soporte usado.

https://tinyurl.com/yxqj4bf5

En resumen, para un análisis verdaderamente preciso sobre la transparencia municipal en España, resulta imprescindible, en primer lugar, un análisis preliminar sobre la ubicación de esa información. En particular, es necesario comprobar dónde se publicita esta información en todo tipo de ayuntamientos, cualquiera que sea su población, como justificamos y señalamos a continuación.

\section{Marco teórico}

A pesar del breve periodo de desarrollo de la ley de transparencia, sin contar con las leyes autonómicas aprobadas en su mayoría a posteriori, son numerosos los estudios sobre transparencia municipal y su cumplimiento realizados:

https://tinyurl.com/y677zf8j

En la tabla 5 se han recogido los trabajos que evalúan la transparencia municipal desde un punto de vista integral, descartando los trabajos que se centraban en aspectos concretos de la transparencia municipal, como la información exclusivamente económica ${ }^{3}$.

\subsection{Principales aspectos de los estudios revisados}

Como se infiere del cuadro, se han elegido cuatro criterios de agrupación de estos estudios. El primero es el rango de población de los municipios analizados, que hemos dividido en cuatro categorías: más de 50.000 habitantes, más de 30.000 habitantes, más de 20.000 habitantes, más de 10.000 habitantes, o todos los tramos de población. Un segundo aspecto es el tipo de ubicación analizada en los mismos, ya sea la de sitios web municipales, de cualquier tipo, que contengan información sobre transparencia, o bien, si se centran en portales de transparencia específicos. En tercer lugar, el ámbito territorial sobre el que se realiza el estudio: si es estatal, si abarcan alguna comunidad autónoma específica, o varias, o si están restringidos a una provincia concreta. Por último, se han revisado las diferentes metodologías empleadas para realizar estos análisis.

En lo que se refiere a la ubicación de la información analizada, diecinueve de los veinticuatro estudios recogidos aquí han analizado las sedes / webs de los ayuntamientos (SWA) y no sus portales de transparencia (PT). Este hecho ha podido llevar a excluir, por ejemplo, a los ayuntamientos que no tienen sede web pero sí portal de transparencia en activo o los que no tienen conectado su portal de transparencia desde su sede web oficial. En este estudio se han encontrado ejemplos de ambos casos.

Puede observarse que apenas hay trabajos referidos al ámbito estatal (Delgado-Jalón; Navarro-Heras; Mora-Agudo, 2017; Rebolledo; Zamora-Medina; Rodríguez-Virgili, 2017), que solo se ocupan de municipios mayores de 10.000 habitantes. Buena parte de los trabajos se aplican sobre comunidades autonómicas específicas. En el caso de las comunidades multiprovinciales, se centran en municipios de cualquier tamaño los estudios sobre Canarias (Comisionado de Transparencia de Canarias, 2015, 2016, 2017, 2018; Trenta; Carrascosa-Puertas, 2015), Aragón (Vadillo-Bengoa; Rodríguez-Breijo; Álvarez-Nobell, 2015) y Cataluña (Moreno-Sardá; Molina-Rodríguez-Navas; Corcoy-Rius, 2013). Los estudios en otras comunidades abarcan solo municipios mayores de 10.000 habitantes, ya sea en Andalucía (Subires-Mancera; Cuartero-Naranjo, 2015; De-Vicente-Domínguez; Vera-Balanza, 2015; Garrido-Rodríguez; Zafra-Gómez, 2017), Castilla-La Mancha (Manfredi-Sánchez et al., 2016), Castilla y León (Cabezuelo-Lorenzo; Rey-García; Tapia-Frade, 2016; Herrero-Gutiérrez et al., 2017), Extremadura (Fernández-Falero et al., 20174) y Galicia (Martínez-Roldán; Piñeiro-Otero; Baamonde-Silva, 2016), así como un estudio comparativo entre las comunidades de Andalucía, Aragón, Canarias, Cataluña, Galicia y Madrid (Moreno-Sardá et al., 2015). Siendo Andalucía y Canarias las comunidades más analizadas. Sobre la Comunidad Valenciana, se ha publicado un reciente estudio, descriptivo y centrado en la participación ciudadana (Paricio-Esteban; Bruno-Carlos; Alonso-Romero; García-Alcober, 2020). 
Tabla 5. Agrupamiento de estudios integrales sobre transparencia municipal publicados entre 2013-2020

\begin{tabular}{|c|c|c|c|c|c|c|c|c|c|c|}
\hline \multirow[b]{2}{*}{$N^{\circ}$ de habitantes } & \multirow[b]{2}{*}{ Estudios } & \multicolumn{2}{|c|}{ Análisis } & \multicolumn{3}{|c|}{$\begin{array}{c}\text { Ámbito } \\
\text { territorial }\end{array}$} & \multicolumn{4}{|c|}{$\begin{array}{l}\text { Metodología de } \\
\text { evaluación }\end{array}$} \\
\hline & & $\sum_{n}^{5}$ & $\stackrel{5}{a}$ & 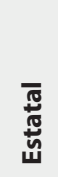 & 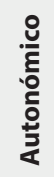 & 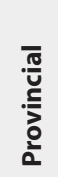 & $E$ & 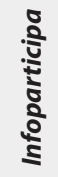 & 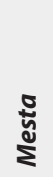 & 气ั \\
\hline \multirow{3}{*}{$>50.000$} & Delgado-Jalón; Navarro-Heras; Mora-Agudo, 2017 & $\mathrm{x}$ & & $x$ & & & & & & $\mathrm{x}$ \\
\hline & Trenta; Carrascosa-Puertas, 2015 & $x$ & & & $\mathrm{x}$ & & & $\mathrm{x}$ & & \\
\hline & Vicente-Domínguez; Vera-Balanza, 2015 & $x$ & & & $x$ & & & $\mathrm{x}$ & & \\
\hline$>30.000$ & Martínez-Roldán; Piñeiro-Otero; Baamonde-Silva, 2016 & $x$ & & & $x$ & & $x$ & & & \\
\hline \multirow{3}{*}{$>20.000$} & Garrido-Rodríguez; Zafra-Gómez, 2017 & & $x$ & & $\mathrm{x}$ & & & & & $x$ \\
\hline & Herrero-Gutiérrez; Ruano-López, 2017 & $x$ & & & $x$ & & & $x$ & & \\
\hline & Moreno-Sardá et al., 2015 & $\mathrm{x}$ & & & $x$ & & & $x$ & & \\
\hline \multirow{7}{*}{$>10.000$} & Cabezuelo-Lorenzo; Rey-García; Tapia-Frade, 2016 & $x$ & & & $\mathrm{x}$ & & & $x$ & & \\
\hline & Fernández-Falero et al., 2017 & $x$ & & & $x$ & & & $x$ & & \\
\hline & Herrero-Gutiérrez et al., 2017 & $x$ & & & $x$ & & & $x$ & & \\
\hline & Manfredi-Sánchez et al., 2016 & $x$ & & & $x$ & & & $x$ & & \\
\hline & Aguilera-Povedano; Matas-Pastor, 2015 & $x$ & & & $x$ & & & $x$ & & \\
\hline & Rebolledo; Zamora-Medina; Rodríguez-Virgili, 2017 & $x$ & & $x$ & & & & $x$ & & \\
\hline & Subires-Mancera; Cuartero-Naranjo, 2015 & $x$ & & & $x$ & & & $x$ & & \\
\hline \multirow{10}{*}{ Todos los tramos } & Alonso-Magdaleno; García-García, 2014 & $x$ & & & $x$ & & $x$ & & & \\
\hline & Comisionado de Transparencia de Canarias, 2015 & & $\mathrm{x}$ & & $x$ & & & & & $\mathrm{x}$ \\
\hline & Comisionado de Transparencia de Canarias, 2016 & & $x$ & & $x$ & & & & $x$ & \\
\hline & Comisionado de Transparencia de Canarias, 2017 & & $x$ & & $x$ & & & & $x$ & \\
\hline & Comisionado de Transparencia de Canarias, 2018 & & $x$ & & $x$ & & & & $x$ & \\
\hline & Moreno-Sardá; Molina-Rodríguez-Navas; Corcoy-Rius, 2013 & $\mathrm{x}$ & & & $x$ & & & $x$ & & \\
\hline & Pagán-Martínez et al., 2018 & $\mathrm{x}$ & & & & $x$ & & & & $x$ \\
\hline & Prieto-Sánchez, 2015 & $\mathrm{x}$ & & & & $\mathrm{x}$ & & $\mathrm{x}$ & & \\
\hline & Vadillo-Bengoa; Rodríguez-Breijo; Álvarez-Nobell, 2015 & $\mathrm{x}$ & & & $x$ & & & $\mathrm{x}$ & & \\
\hline & Vera-Balanza; López-Franco, 2015 & $x$ & & & & $x$ & & $x$ & & \\
\hline & & 19 & 5 & 2 & 19 & 3 & 2 & 15 & 3 & 4 \\
\hline & & \multicolumn{2}{|c|}{24} & \multicolumn{3}{|c|}{24} & \multicolumn{4}{|c|}{24} \\
\hline
\end{tabular}

En cuanto a análisis sobre comunidades uniprovinciales o provincias específicas, aparecen referencias a Asturias (Alonso-Magdaleno; García-García, 2014), Baleares (Aguilera-Povedano; Matas-Pastor; Durán-Mañes, 2015), Madrid (Herrero-Gutiérrez; Ruano-López, 2017), Cádiz (Vera-Balanza; López-Franco, 2015), Granada (Prieto-Sánchez, 2015) y Albacete (Pagán-Martínez et al., 2018) .

Con respecto a la metodología de análisis sobre el contenido de los sitios analizados, se han detectado distintas herramientas de análisis y evaluación. Infoparticipa es la metodología mayoritaria de análisis y evaluación, si bien no en todos los casos se emplea el mismo número de items analizados. Otras metodologías minoritarias, pero usadas en más de un estudio son: Índice de Transparencia y Acceso a la Información (ITA) (hasta 2016), Metodología de evaluación y seguimiento de la transparencia (Mesta) (empleada en versión adaptada, Índice de Transparencia de Canarias, a la Ley 12/2014 de transparencia de Canarias, en todos los informes del Comisionado de transparencia autonómico menos el primero de 2015).

Otras metodologías de análisis y evaluación detectadas son la usada por Garrido-Rodríguez; Zafra-Gómez (2017, pp. 88-89), realizada en colaboración con la Cámara de Cuentas de Andalucía y la Fundación Centro de Estudios Andaluces. La fundamentada en los servicios básicos online que los ayuntamientos deben ofrecer a los ciudadanos según las directrices Plan de Acción de la eEurope 2005 y Plan de Actuación i2010 (Pagán-Martínez et al., 2018, pp. 180-181). Y, por último, el Test de aplicación municipal de la Ley de Transparencia desarrollado por Delgado-Jalón; Navarro-Heras; Mora-Agudo (2017, p. 114).

Solo Canarias tiene un $100 \%$ de portales de transparencia con contenido 
Las diferentes metodologías de análisis y evaluación muestran, a nuestro juicio, que estamos lejos aún de poder hablar de evaluación de la transparencia municipal en España en términos generales. La ley nacional establece unos mínimos, que han de ser desarrollados luego por las leyes autonómicas, y no lo hacen de la misma manera. A esas dos normativas territoriales hay que añadir las propias que han llevado a cabo muchos municipios. No obstante, esta discusión metodológica, tanto cualitativa como cuantitativa, queda fuera del alcance de este artículo.

\subsection{Necesidad de un estudio preliminar de ubicación de la información municipal de transparencia}

Así, se hacía necesario, a nuestro juicio, realizar un análisis preliminar sobre estas ubicaciones, a modo de radiografía, sobre la presencia de sitios web dedicados a la transparencia municipal en toda España, al menos, para dar cuenta de su mera existencia y, secundariamente, para detectar de forma básica si esa presencia era meramente formal, es decir, si dichas ubicaciones tenían realmente contenidos, por mínimos que fueran.

Por otra parte, aunque diez estudios incluyen los municipios de menos de 10.001 habitantes, sólo se analizan los municipios de este rango de población en trece provincias. De esas trece, sólo Albacete pertenece a una de las dos CCAA, Castilla-La Mancha y Castilla y León, que reúnen el 39\% (3.167) de todos los municipios españoles (8.131) y el 51.6\% (3.108) de los municipios españoles de hasta 10.000 habitantes (6.026) (INE, 2020).

Este análisis preliminar requería ser, por lo tanto, exhaustivo e independiente de cual fuera la población de los municipios. Más aún, debía centrarse sobre todo en los municipios más pequeños, los grandes olvidados de los estudios; y también debía de abarcar, en lo posible, cuantas formas diferentes de presentar esa información sobre transparencia: sitios web municipales y portales de transparencia y su tipología: propia, provincial, autonómica o estatal, se están dando en la realidad y en qué porcentajes.

Dado el enorme número de municipios en España, se eligió partir de una muestra estatal estratificada de los municipios. Además, se tuvieron en cuenta dos elementos clave: la presencia de municipios de todas las provincias españolas, por un lado, y, por otro, la distribución territorial a nivel provincial, esto es, los rangos de población de municipios que contiene cada provincia. Ambas cuestiones se detallan en el apartado 3.1. Por citar algunos ejemplos, las provincias pertenecientes a comunidades como Castilla y León, así como Castilla-La Mancha, concentran un gran número de municipios con bajos rangos de población (menores de 1.000 habitantes), mientras que hay provincias, como Cádiz, en las que no existen municipios de esos tamaños.

\section{Metodología}

El trabajo presentado es una investigación descriptiva en la que se muestra el estado actual de los medios usados por los municipios españoles para hacer su transparencia, así como su localización y su vinculación con las webs/sedes web oficiales de los mismos. El objetivo general es, por tanto, detectar dónde están realizando la publicidad activa los municipios españoles en todos los tramos de población definidos por el Instituto Nacional de Estadística (INE, 2020) del territorio español. Dicha información puede estar en la propia web del municipio, en portales específicos de transparencia, o en la sede electrónica, tal como recogen las distintas normativas. Consecuentemente, las variables analizadas han sido:

(i) Presencia de sede web en el ayuntamiento (SWA).

(ii) Presencia de portal de transparencia (PT).

(iii) Ubicación de PT. En sede propia, o en sede proporcionada por otra administración.

(iv) Enlace al PT desde la SWA.

(v) Presencia de información en el PT, o si están vacíos. Se ha considerado como vacío el portal que tenía menos de 10 items de información, independientemente de su actualización.

En este trabajo se ha definido el "ítem de información" como la información que ha de ser publicitada por las administraciones públicas, según algunas de las leyes o normas estatales o autonómicas vigentes, y que está recogida en alguna de las categorías o apartados de la SWA o del PT.

Para la localización del espacio destinado a la transparencia de los 1.955 municipios de la muestra se han hecho búsquedas por SWA, por transparencia y por la sede electrónica (SE) del ayuntamiento. Se repitieron las tres búsquedas en la mayor parte de los municipios de la muestra, lo que dio como resultado la localización de municipios con dos PT, y el aumento del número de PT analizados.

\subsection{Muestra}

El universo de discurso a enero de 2020 es de 8.131 municipios en España. El tamaño de la muestra se ha determinado con un coeficiente de confianza del $99,7 \%$ y un porcentaje de error del $3 \%$, siendo el tamaño final de la muestra analizada de 1.955 municipios.

Con la finalidad de que todos los tramos poblacionales y territorios estuviesen adecuadamente representados, se usaron dos criterios complementarios para la elaboración de la muestra:

i) Tramos. Se estratificó según todos los tramos contemplados por el INE (desde el más pequeño, de 0 a 100 habitantes, hasta el mayor, de más de 500.000 habitantes). Se añadió un tramo no recogido en el INE en el tramo-estrato de 50.001 


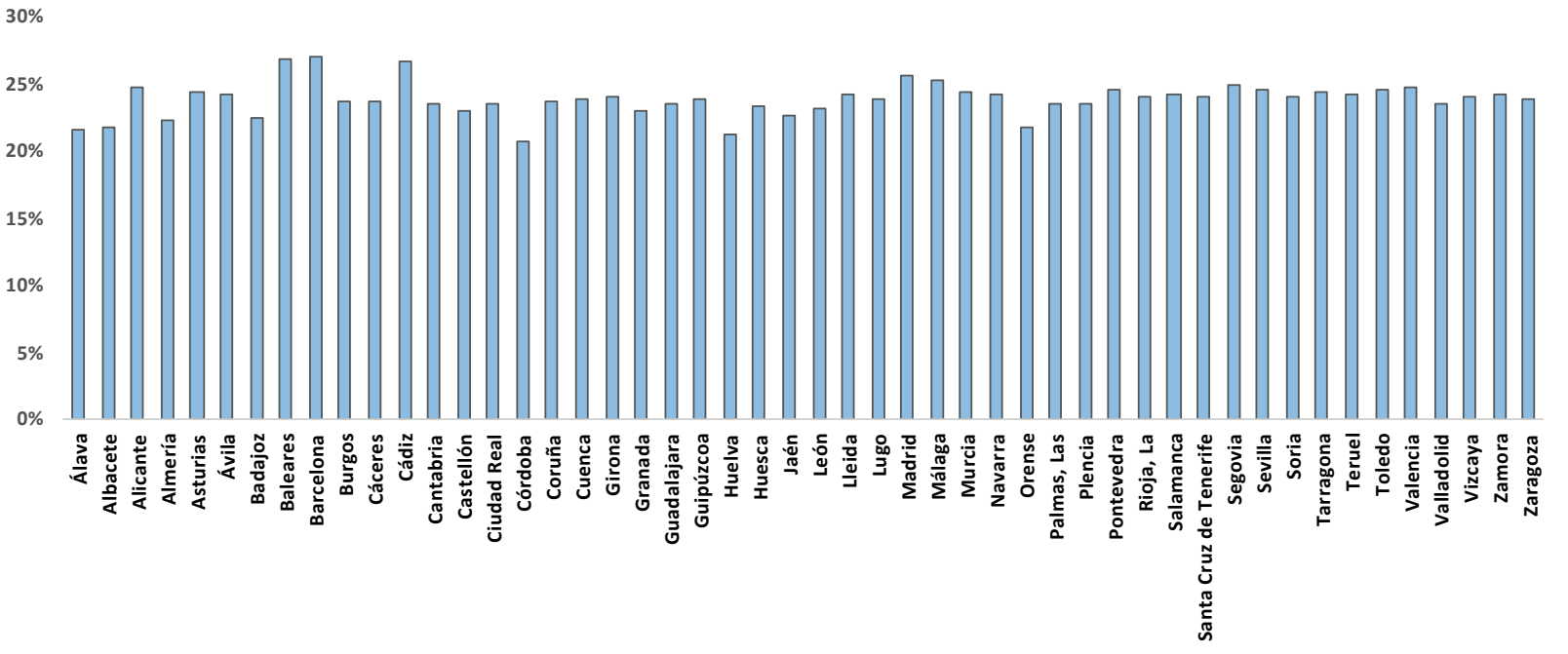

Gráfico 1. Distribución porcentual de la muestra por provincias

a 100.000 habitantes: 50.001- 74.999 y 75.000-100.000, para poder obtener información de los municipios de más de 75.000 habitantes con calificación de gran población en relación a los del mismo tramo que no la tienen.

ii) Provincias. Este criterio de distribución de la muestra por tanto por ciento de estratos poblacionales se conjugó, además, con la distribución por provincias; de manera que el número de municipios de ninguna provincia quedara por debajo del $20 \%$ de representación en el universo de discurso, ni por encima del $30 \%$, garantizando así que todas se encontrarían adecuadamente representadas (gráfico 1). Se añadieron, además, las ciudades autónomas de Ceuta y Melilla.

Para la asignación de municipios a la muestra, se ordenaron aleatoriamente todos los municipios españoles mediante un código numérico. De ahí se extrajeron, para cada rango y provincia, los primeros 1.955 municipios por orden de código.

Seguidamente, se revisó la muestra, comprobando que no hubiera, para ninguna provincia, más del $20 \%$ ni menos del $30 \%$ de los municipios en cada rango y en cada provincia. No obstante, se tuvieron en cuenta las provincias que no tenían municipios en algún rango (por ejemplo: Almería no tiene municipios con población menor a 101 habitantes), provincias que estaban infrarrepresentadas (no se cubría el 20\% del total de sus municipios en su rango) o que, al contrario, estaban sobrerrepresentadas (más del $30 \%$ de su rango). Se comprobaron una a una, realizando las reasignaciones mínimas para asegurar la representación de todas las provincias y rangos, así como de la muestra en conjunto.

Una vez aplicados ambos criterios, la distribución final de la muestra fue la recogida en la tabla 6.

Tabla 6. Distribución de la muestra en estratos. Fuente: INE, 2020.

https://tinyurl.com/yxyg $477 q$

\begin{tabular}{|c|c|c|c|c|}
\hline $\begin{array}{c}\text { Tramos-estrato del } \\
\text { estudio }\end{array}$ & $\begin{array}{l}\text { No de municipios en } \\
\text { tramo-estrato }\end{array}$ & $\%$ del tramo-estrato & $\begin{array}{l}\text { No de municipios por } \\
\text { tramo-estrato según } \%\end{array}$ & $\begin{array}{l}\text { No de municipios } \\
\text { después de aplicar el } \\
\text { criterio por provincia }\end{array}$ \\
\hline $0-100$ & 1.352 & 16,6 & 325 & 326 \\
\hline $101-500$ & 2.645 & 32,5 & 636 & 638 \\
\hline $501-1.000$ & 1.003 & 12,3 & 241 & 240 \\
\hline $1.001-2.000$ & 877 & 10,8 & 211 & 211 \\
\hline $2.001-3.000$ & 478 & 5,9 & 115 & 112 \\
\hline $3.001-5.000$ & 474 & 5,8 & 113 & 114 \\
\hline $5.001-10.000$ & 549 & 6,8 & 133 & 132 \\
\hline $10.001-20.000$ & 340 & 4,0 & 78 & 81 \\
\hline $20.001-30.000$ & 158 & 1,9 & 37 & 40 \\
\hline $30.001-50.000$ & 107 & 1,3 & 25 & 24 \\
\hline $50.001-74.999$ & 48 & 0,6 & 11 & 11 \\
\hline $75.000-100.000$ & 37 & 0,5 & 10 & 11 \\
\hline $101.000-500.000$ & 57 & 0,7 & 14 & 14 \\
\hline$>500.000$ & 6 & 0,1 & 1 & 1 \\
\hline Total & 8.131 & 99,8 & 1.950 & 1.955 \\
\hline
\end{tabular}




\section{Resultados del análisis}

\subsection{Presencia de sedes web municipales}

El total de municipios que no tienen sede web del ayuntamiento (SWA) en toda la muestra es de 266 (13,6\%). Se concentran en los cinco tramos de menor población. Por tramos varía, yendo del 37,12\% del tramo más pequeño (0-100), hasta el 1,79\% del tramo de 2.001-3.000 habitantes.

Los municipios que están haciendo publicidad activa en la SWA son 38 de los 1.955; de ellos, 15 no tienen portal de transparencia (PT) (0,77\%). En su mayor parte, pertenecen a los tramos de menos de 10.000 habitantes, y alguno hasta el tramo 20.000-30.000. La mayoría se concentran en el País Vasco, aunque se han encontrado casos aislados también en Andalucía, Aragón, Baleares, Cantabria, Castilla-La Mancha y Navarra.

\subsection{Presencia de los portales de transparencia}

Se han detectado casos de municipios que, a pesar de no tener SWA, tienen PT. Son 197 de los municipios de la muestra (10\%). Si tenemos en cuenta los que, a pesar de no tener PT, sí que están haciendo publicidad activa en su SWA (el anterior $0,77 \%)$, el porcentaje de municipios analizados que no ofrecen transparencia por ninguna de las opciones previstas sería el 9,23\%. Ello indica que parece estar penetrando más la transparencia que la SWA. No obstante, son, en este caso, los dos tramos de población más pequeños, 0-100 y 101-500, los que concentran los porcentajes más altos con $16,36 \%$ y $13,32 \%$, respectivamente.

Debe hacerse mención a la existencia de casos con duplicidad (municipios con dos PT), lo que eleva a 1.971 el total de PT localizados y analizados. Estas duplicidades se concentran en los nueve tramos más pequeños. No obstante, se han encontrado dos casos más: uno, en el tramo 30.00150.000 , y el otro, en el 10.000150.0000. Por lo tanto, el número total de municipios con dos PT es de 213 , el $11 \%$. El uso de dos portales está siendo muy distinto: municipios que están usando ambos para poner distintos tipos de información; otros han usado uno de ellos hasta un año, y el otro, a partir del siguiente; en algunos casos, los dos están vacíos o tienen poca información, y, en otros tienen la misma información.

\subsection{Ubicación de los portales de transparencia: en sitio propio o en sitio proporcionado por otra administración}

De los 1.971 encontrados, el 71,64\% (1.412) se encuentran en sede electrónica (SE). El 3,5\% (69) usan el PT del Minhap para las entidades locales, gob.es.

Los PT elaborados por los propios municipios suponen el $14,61 \%$ de los analizados, habiendo en todos los tramos, aunque concentrados fundamentalmente en los de mayor población. El 0,46\% de los PT analizados se encuentra en iniciativas comarcales, o similares, todos en los tres tramos poblacionales más pequeños.

En el caso de las iniciativas de diputaciones provinciales, las dieciséis

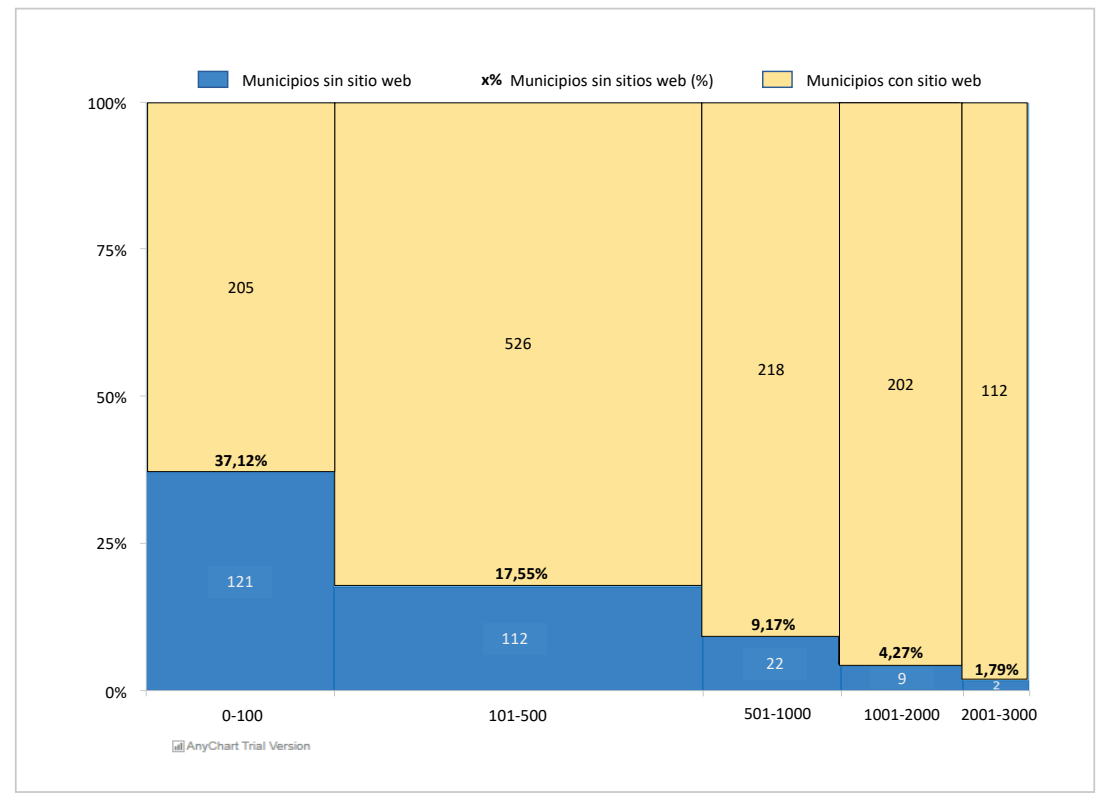

Gráfico 2. Número y porcentaje en tramo de municipios que no tienen sede web

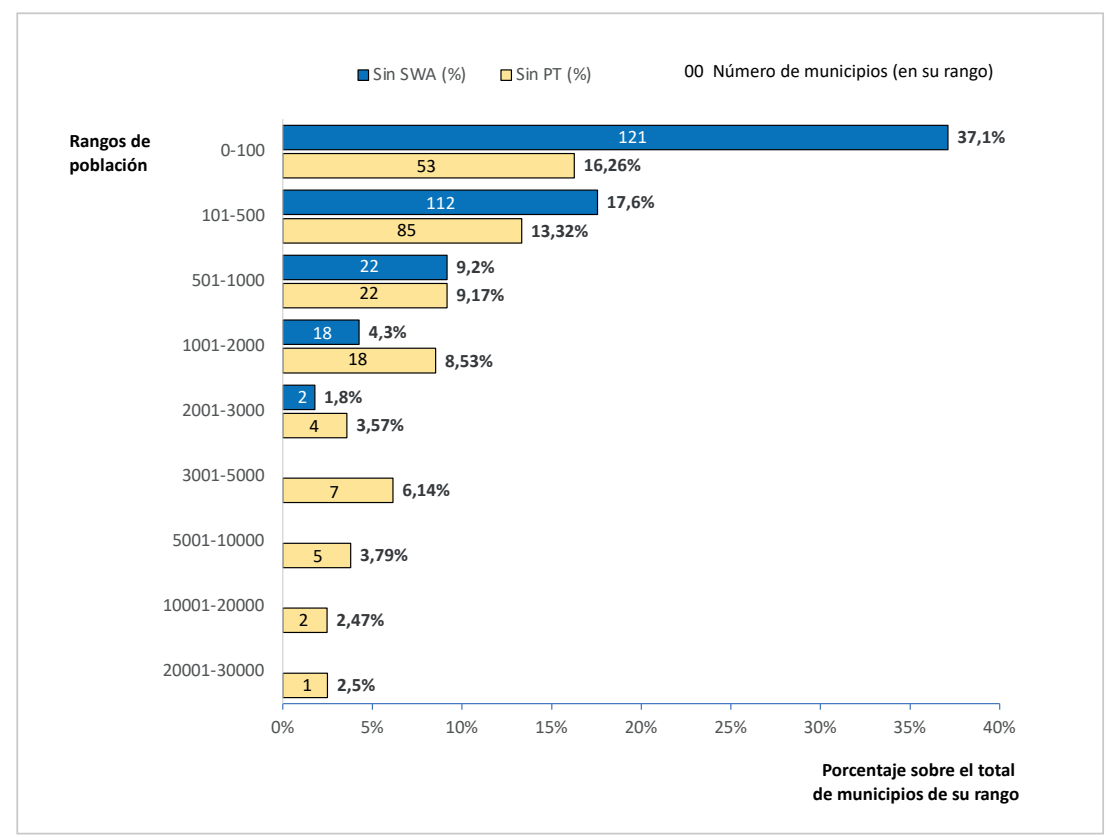

Gráfico 3. Número y porcentaje en tramo de municipios que no tienen sitio web o portal de transparencia 
encontradas suponen el 8,02\% de los PT analizados, desde el tramo más pequeño hasta el de 30.00150.000: Almería, Álava, Badajoz, Cáceres, Cádiz, Córdoba, A Coruña, Granada, Guipúzcoa, Huelva, Lugo, Málaga, Orense, Salamanca, Sevilla y Vizcaya.

El $1,78 \%$ de los PT analizados se encuentran en tres proyectos autonómicos: Aragón (la mayoría), Comunidad Valenciana y Galicia.

De nuevo, la casuística es muy variada en los casos de municipios con dos PT. Muchos de los duplicados tienen una de las plataformas estatal, y otra, autonómica o provincial, aunque también hay casos en los que de los dos, uno es propio. Hay casos que tienen los dos PT estatales, ambos vacíos, ambos con información, etc.

En el 14,61\% de los PT que se han contabilizado como propios por encontrarse en SWA, se pueden encontrar casos en los que el diseño no es del ayuntamiento sino de la diputación provincial. Esto es, hay diputaciones provinciales que han puesto a disposición de los ayuntamientos de su provincia un diseño de PT para uso en SWA: Almería, Badajoz, Cáceres, Córdoba, Huelva, Jaén, León, Sevilla y Valencia. Se han registrado como propios porque el mantenimiento del PT recae en el ayuntamiento.

Mención aparte merece la distribución por sedes electrónicas, puesto que es la ubicación más usada para el portal de transparencia en la mayoría de tramos. De los PT analizados, el 1,01\% (20) tienen sede electrónica propia (SEP), y el mismo porcentaje y número lo tienen en sede electrónica de su diputación provincial (SEDP). El 12,13\% (239) lo tienen en sede electrónica de su comunidad autónoma (SECA), y están ubicados en municipios de Cataluña y Andalucía. En el caso de Cataluña son casi la totalidad, en el de Andalucía, sólo algunos municipios de Jaén. Del 71,64\% que están usando SE, el 57,03\% (1.124) de los PT analizados se encuentran en sede electrónica estatal (SEE) (gráfico 6).

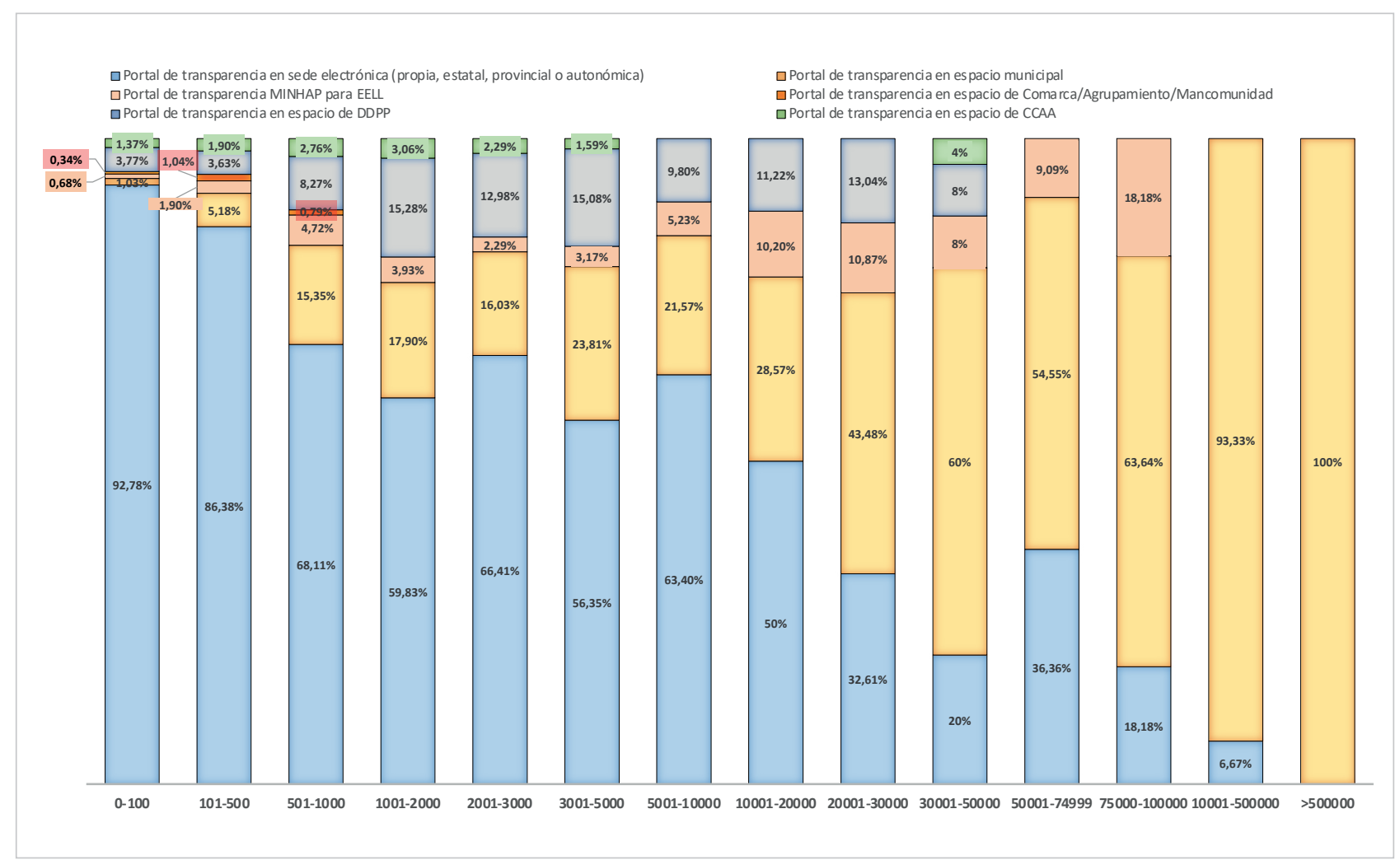




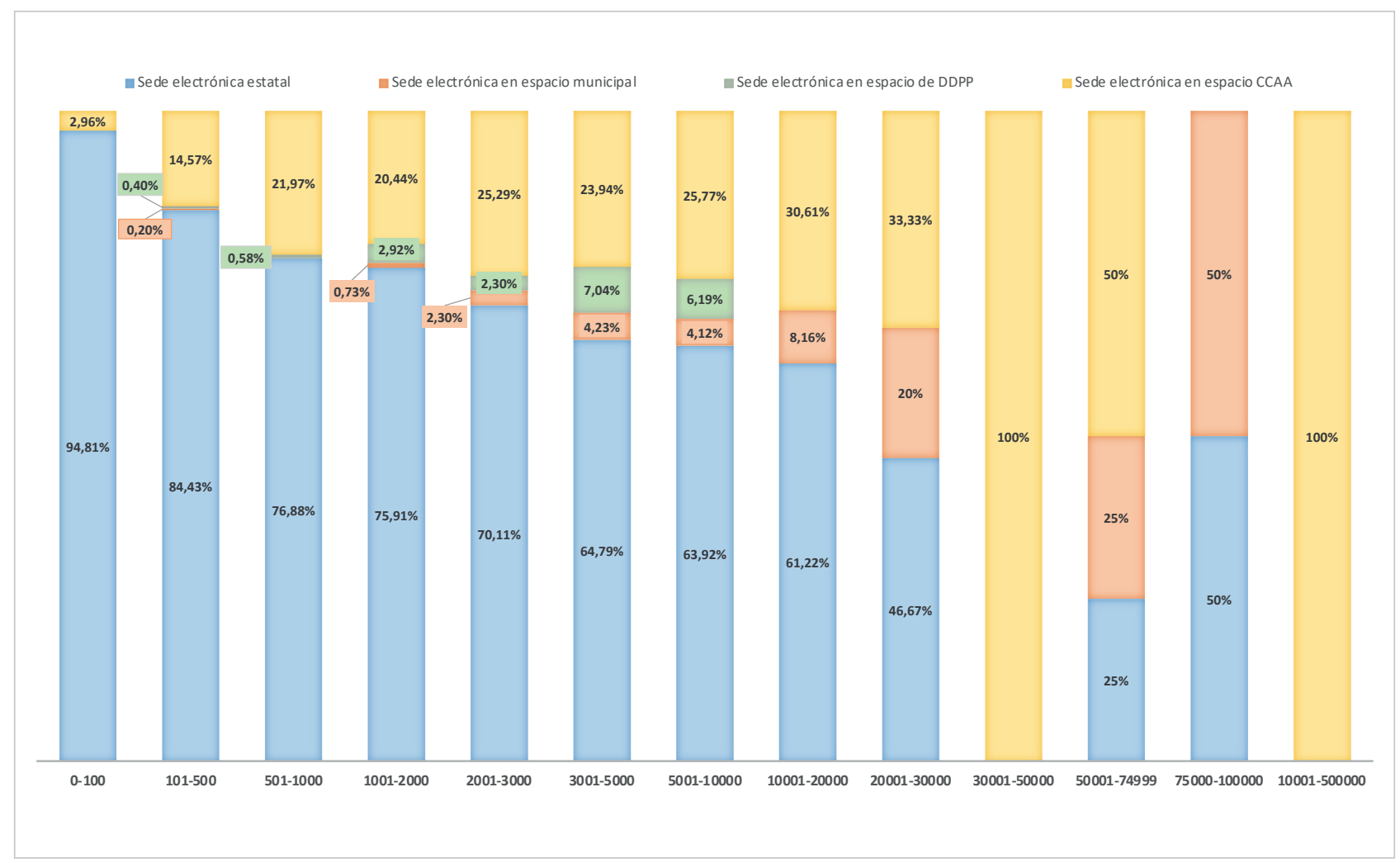

Gráfico 6. Distribución por porcentajes de portales de transparencia por sedes electrónicas según administración

\subsection{Enlace al portal de transparencia desde la sede web municipal}

Dado que existe un porcentaje significativo de municipios con dos portales de transparencia (11\%), llegando a alcanzar en algunos tramos más del $20 \%$ de los municipios analizados, se presentarán los resultados de los primeros y segundos portales de transparencia separados. En los casos de municipios con dos portales, se eligió como primer portal el que estuviera enlazado; si ambos lo estaban o ninguno lo estaba, entonces se usaron distintos criterios en este orden: i) el de diseño o ubicación propios; ii) si los dos estaban enlazados, el enlazado desde transparencia, o equivalentes (gobierno abierto, por ejemplo); iii) en el caso de que ninguno lo estuviera, el que tuviese contenido; iv) si ambos tenían contenido, pero ninguno enlazado: ubicación estatal (si ambos eran de ubicación estatal, el portal de transparencia local); ubicación autonómica; ubicación provincial (si ambos eran provinciales, el que más información contuviese) (gráficos 7 y 8).

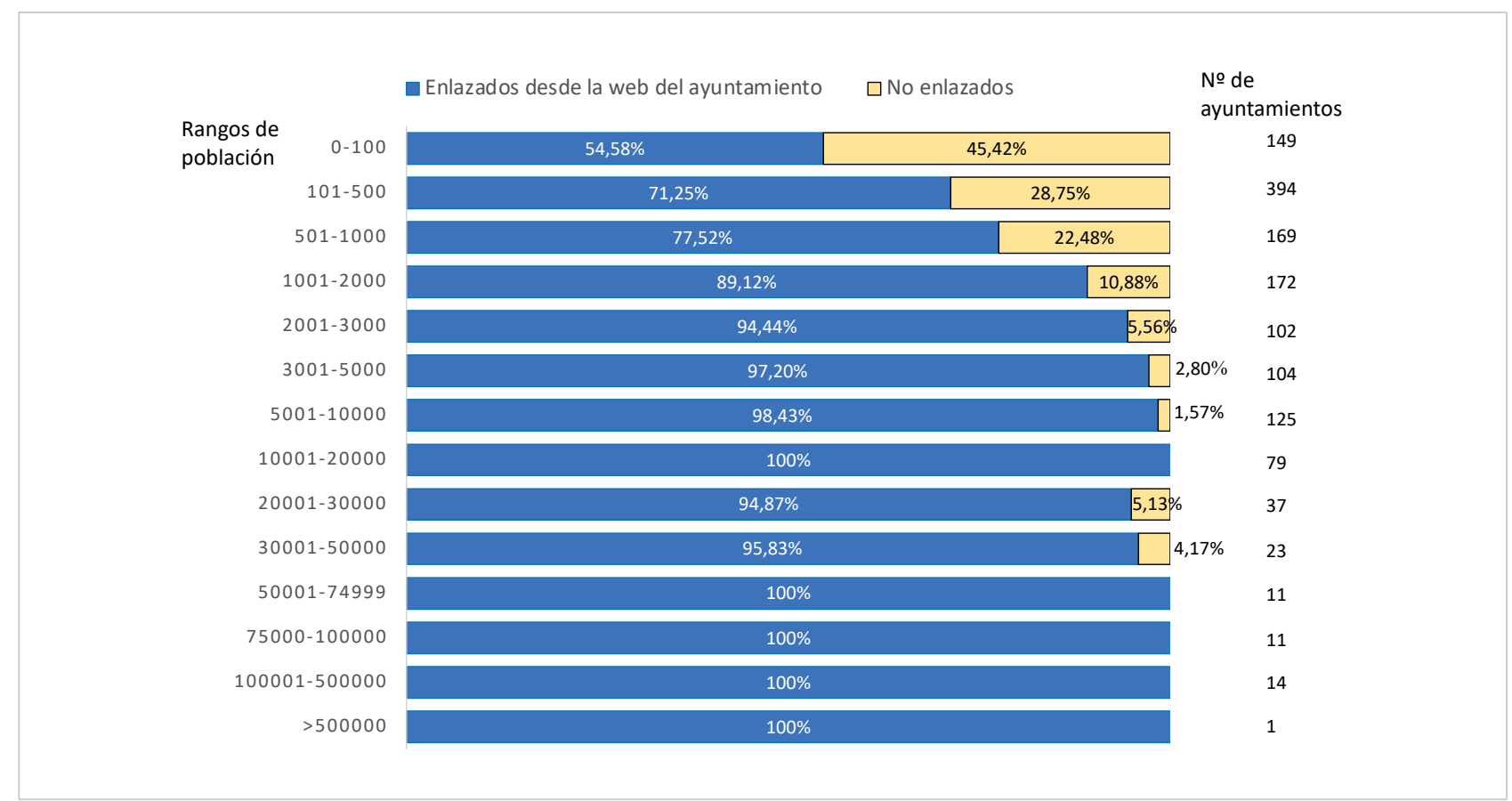

Gráfico 7. Número y porcentaje en tramo de primeros PT enlazados desde la sede web del ayuntamiento 


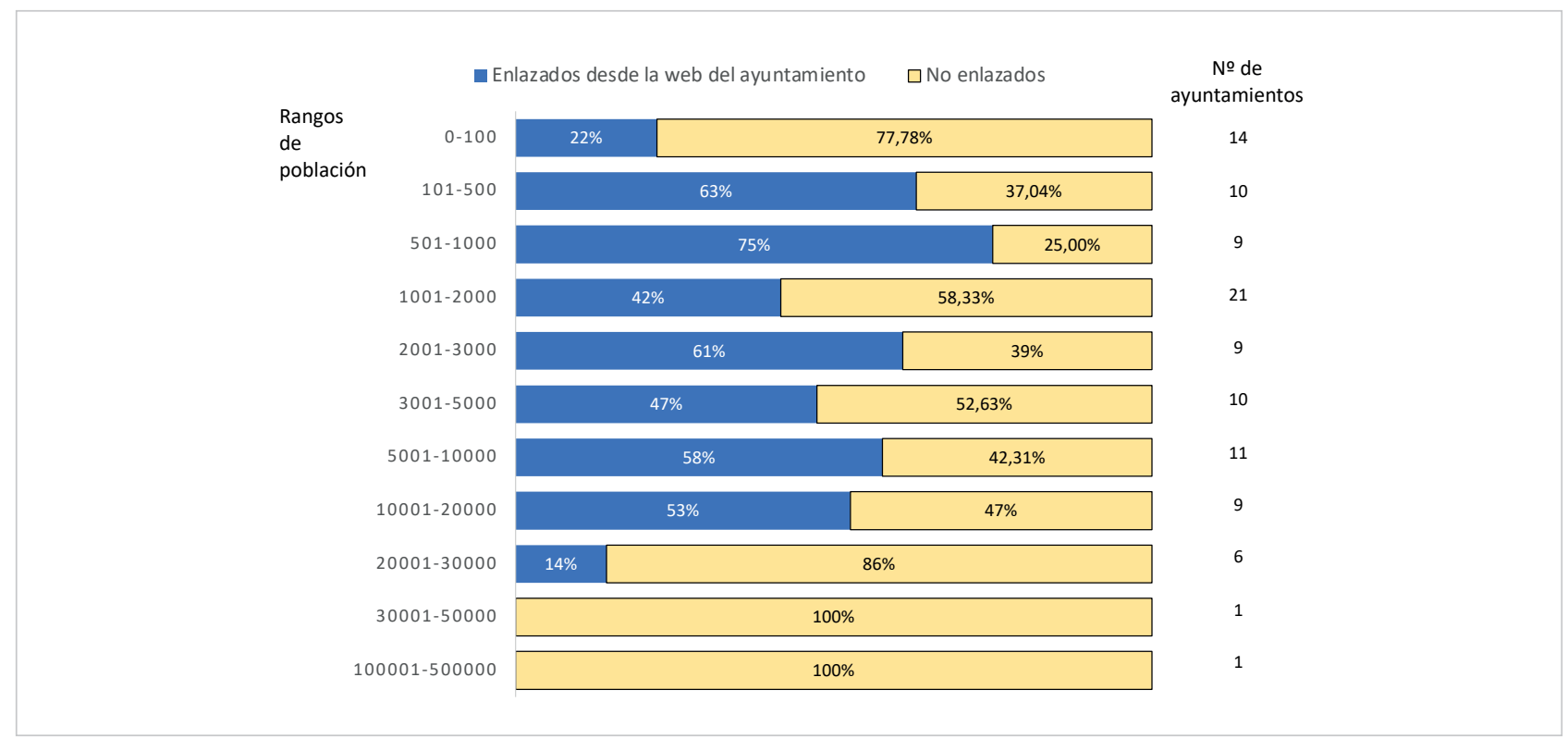

Gráfico 8. Número y porcentaje en tramo de segundos PT enlazados desde la sede web del ayuntamiento

\subsection{Presencia de información en el portal, o si están vacíos}

El segundo de los grandes problemas detectados es la cantidad de PT que están vacíos. Se ha considerado como vacío el portal que tenía menos de 10 items de información, tal como se definió el concepto "ítem de información" en el apartado 3. En el caso de los primeros PT en muestra, 764 están vacíos, el 39,08\%, concentrándose en este caso los porcentajes más elevados en los tramos 0-100 y 2.001-3.000, con el 78,75\% y el 78,85\%, respectivamente (gráfico 9).

En el caso de los segundos PT, son 99, el 46,48\%, los que están vacíos. Siendo en este caso los porcentajes más altos los de los tramos $0-100$ y $101-500$, con $88,9 \%$ y $81,48 \%$, respectivamente (gráfico 10 ).

El porcentaje total de primeros y segundos PT en muestra que están vacíos asciende al 44,14\%.

Por otra parte, aunque la mayoría de los 1971 primeros y segundos portales encontrados funcionan, existen quince que no funcionan (tabla 7 ), el $0,76 \%$, que se concentran todos en los denominados primeros PT, funcionando todos los segundos portales. No es un porcentaje significativo, pero sí lo son sus consecuencias: esos municipios no están haciendo publicidad activa, incluso aunque ofrezcan el enlace al PT desde su SWA.
Tabla 7. Municipios con primer portal de transparencia que no funciona

\begin{tabular}{|l|c|c|c|c|}
\hline \multicolumn{1}{|c|}{ Tramo } & $\begin{array}{c}\text { Municipios } \\
\text { en el tramo }\end{array}$ & Primer PT & $\begin{array}{c}\text { No } \\
\text { funciona }\end{array}$ & $\begin{array}{c}\% \text { no } \\
\text { funciona }\end{array}$ \\
\hline $0-100$ & 326 & 273 & 7 & 2,56 \\
\hline $101-500$ & 638 & 553 & 1 & 0,18 \\
\hline $501-1.000$ & 240 & 218 & 4 & 1,83 \\
\hline $1.001-2.000$ & 211 & 193 & 1 & 0,52 \\
\hline $3.001-5.000$ & 114 & 107 & 1 & 0,93 \\
\hline $5.001-10.000$ & 132 & 127 & 1 & 0,79 \\
\hline
\end{tabular}

— Primer PT vacío $\square$ Primer PT con contenido

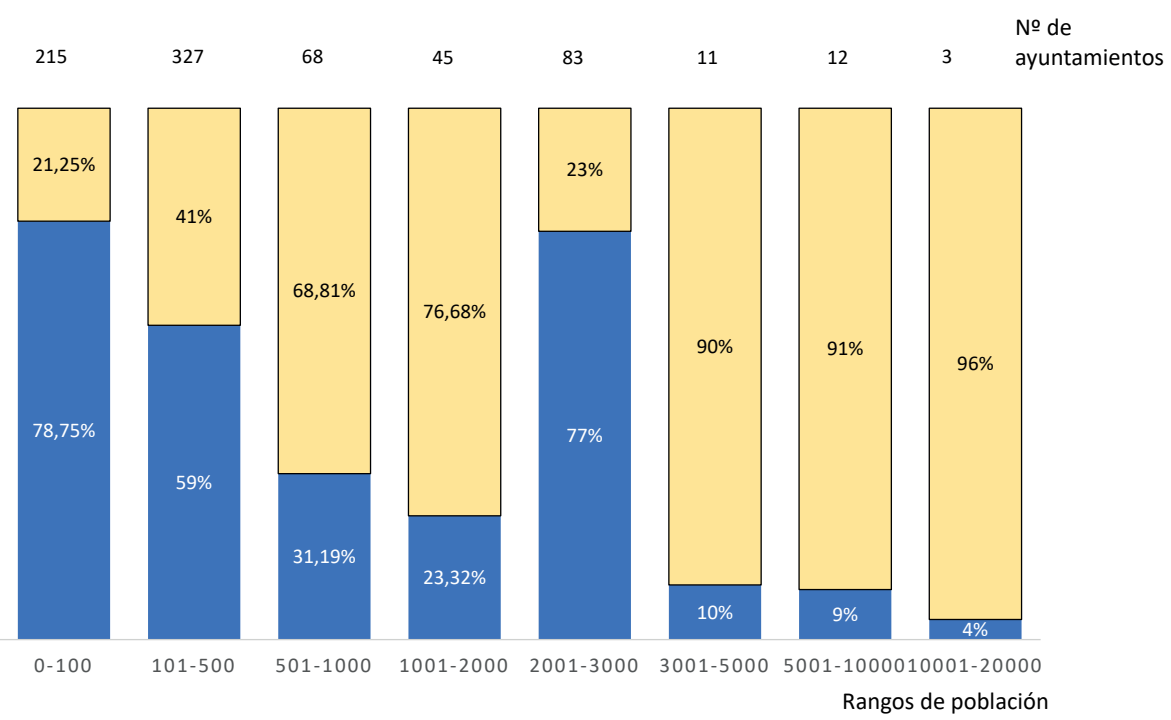




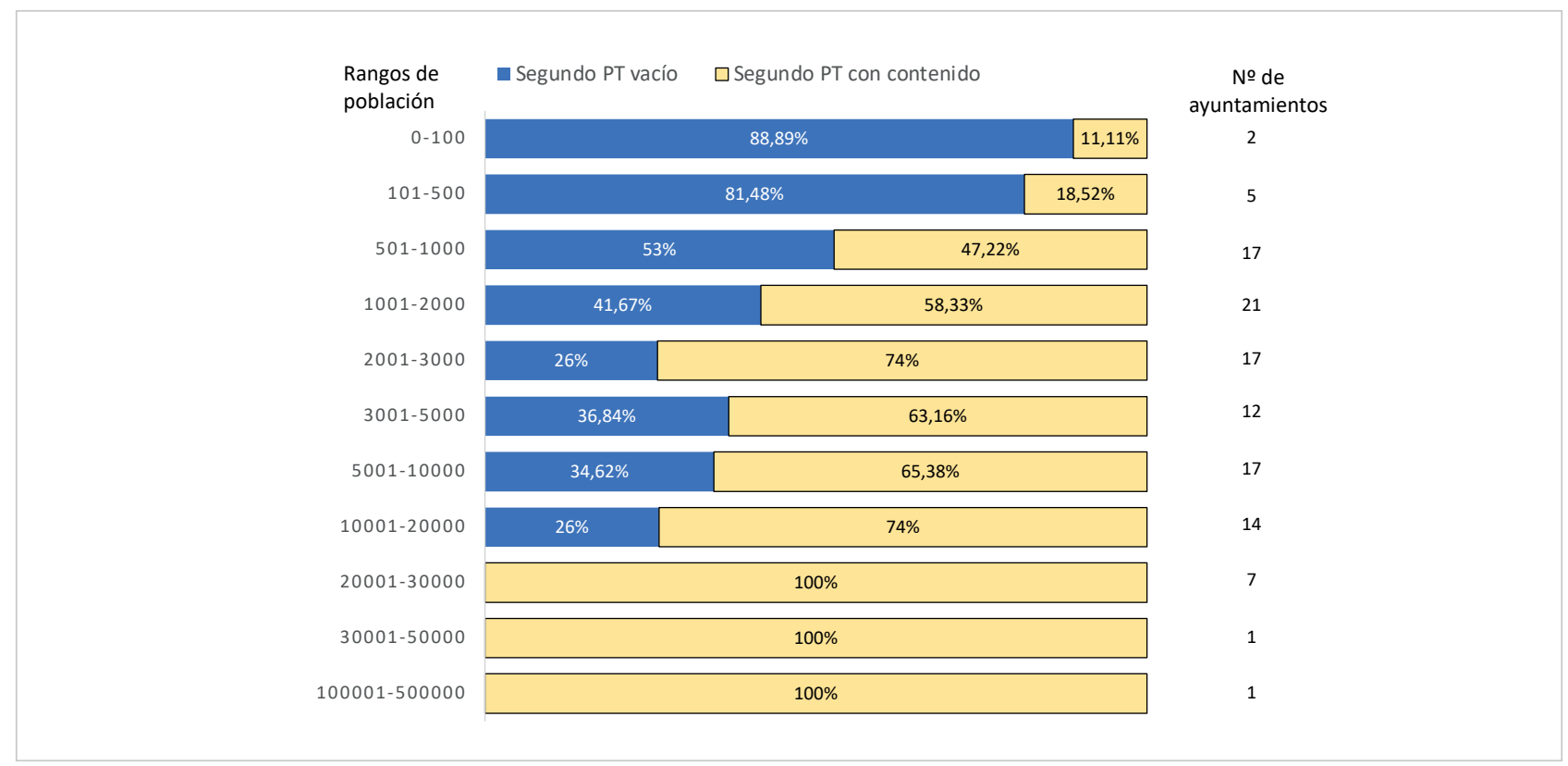

Gráfico 10. Número y porcentaje en tramo de segundos PT vacíos

\section{Conclusiones}

En el caso de la sede web del ayuntamiento (SWA) (variable i), a pesar de que se ha observado que son fundamentalmente las diputaciones provinciales las que dan este soporte a la mayoría de los municipios de los tramos más pequeños, sigue habiendo un $13,6 \%$ de ayuntamientos de la muestra que no tienen SWA. En el caso de los municipios menores de 100 habitantes, se eleva al 37,12\%.

Por otra parte, de los 1.955 municipios analizados, se han localizado 1.971 portales de transparencia (variable ii). El 11\% de municipios analizados tienen dos portales de transparencia (PT); y, al contrario, hay un 9,23\% de municipios que no tienen PT ni tampoco hacen publicidad activa en su SWA. Además, el 39,08\% de los primeros PT están vacíos (variable v). Asimismo, un $0,76 \%$ de los primeros PT no funcionan. Si tenemos en cuenta estos tres conjuntos, el $49,07 \%$ de los municipios analizados no están haciendo publicidad activa. Estos tres factores se concentran, fundamentalmente, en los tramos más pequeños.

La mayoría de los municipios que no tienen portal, o está vacío o no funciona, se concentra en los tramos de población menores de 20.000 habitantes. Esto es, en los ocho tramos de menor población, que se corresponden con el $95 \%$ de los ayuntamientos y el 30,5\% de la población española.

Puesto que en este estudio se han contabilizado como portales con contenido los que tuvieran un mínimo de 10 items de información (variable v), el porcentaje de municipios que no hacen publicidad activa podría ser mucho mayor en los tramos poblacionales más pequeños, en los que, en muchos casos, estaban o pasaban escasamente de ese número de items de información, o tenían la información desactualizada.

\subsection{Sobre la ubicación de los portales de transparencia}

En lo que se refiere a la ubicación (variable iii), el 71,64\% de los 1.971 PT analizados están localizados en una sede electrónica (SE) y el $28,36 \%$ en portales específicos de transparencia. Un pequeño porcentaje, el 0,77\%, muestra su información sobre transparencia directamente en su SWA.

La SE estatal es la más usada con un 57,03\%. y, en segundo lugar, con el 12,13\%, la sede electrónica autonómica (SEA); en este último caso, en su mayor parte son municipios de Cataluña y, unos pocos, de Andalucía, todos en la provincia de Jaén. Un 1,01\% lo están haciendo en SE propia, la mayoría en Santa Cruz de Tenerife y alguno en otras provincias (Barcelona, Castellón y Navarra), y el mismo porcentaje en SE de la diputación provincial, todos en A Coruña.

De las iniciativas de PT en CCAA, solo el 1,73\%, se han detectado únicamente tres: Aragón (cumpletransparencia), Comunidad Valenciana y Galicia (eidolocal). La iniciativa de Aragón, aunque desarrollada por una empresa privada, ha sido adoptada por muchos municipios aragoneses de las tres provincias, por eso se ha considerado de carácter autonómico. Se ha encontrado también en casos aislados de municipios, la mayoría de Cantabria.
El 49,07\% de municipios analizados no está haciendo publicidad activa 
Las iniciativas de PT en diputaciones provinciales, que son el $8,02 \%$ de la muestra, aparecen en dieciséis provincias: Almería, Álava, Badajoz, Cáceres, Cádiz, Córdoba, A Coruña, Granada, Guipúzcoa, Huelva, Lugo, Málaga, Orense, Salamanca, Sevilla y Vizcaya. También se han encontrado municipios que usan en su SWA el diseño del PT municipal de su diputación: Almería, Badajoz, Cáceres, Córdoba, Huelva, Jaén, León, Sevilla y Valencia.
Solo Cádiz, A Coruña, Lérida, Málaga, Las Palmas y Santa Cruz de Tenerife alcanzan el $100 \%$ de portales de transparencia con contenido

Se ha podido constatar, pues, que las administraciones públicas de los tres niveles, y superiores al municipio, están haciendo un esfuerzo por dar soporte para la transparencia a los municipios de menor población.

A pesar de todos estos esfuerzos, a día de hoy, sólo seis territorios provinciales presentan un $100 \%$ de PT en la muestra analizada. La primera provincia es Lérida, cuyos portales están en la iniciativa autonómica en SEA, e incluye a municipios de casi todos los tramos de la muestra, menos de 0-100. También se encuentran en este grupo las dos provincias canarias, Las Palmas y Santa Cruz de Tenerife: los municipios cuentan con un seguimiento anual del Comisionado de Transparencia autonómico, pero los portales de transparencia proceden de iniciativas diversas. Las provincias que responden mayoritariamente a iniciativas provinciales son A Coruña y Málaga: la primera en sede electrónica de la diputación provincial (SEDP), la segunda en la iniciativa de PT de la diputación. La sexta y última provincia es Cádiz (sin municipios en los tramos $\leq 1.000$ habitantes), en la que no se ha podido identificar ni soporte ni seguimiento de la diputación, incluyendo entre su tipología: portales municipales propios, SEE y PT del Minhap para entidades locales (EELL).

Así, todo parece indicar que, solo si los ayuntamientos aceptan bien del diseño y mantenimiento de la herramienta usada (A Coruña, Lérida y Málaga) bien del seguimiento de la publicidad activa (Comisionado de Transparencia en las dos provincias canarias), hay una repercusión positiva en la transparencia municipal de los tramos poblacionales de ayuntamientos de menor población.

Por último, no se han observado diferencias en lo relacionado con la presencia y ubicación del PT, y demás variables analizadas, entre los municipios de 75.000-100.000 con calificación de gran población y los que no la tienen.

\subsection{Principales problemas detectados}

En cuanto a los principales problemas detectados, el primero es la falta de publicidad en SWA sobre dónde se encuentra el PT, además de los casos de municipios con PT y sin SWA. En los municipios con más de un PT, muchos no se encuentran enlazados desde SWA: a veces, son los primeros PT, y en otros casos, ninguno de los dos (SE y apartado de transparencia, por ejemplo). El caso más repetido es el de los PT que se encuentran en SE (variable iv). En el 71,64\% de los PT en SE, se han encontrado muchos casos en los que no se anuncia que, desde el apartado por el que se accede a la SE (sede electrónica, administración electrónica, e-administración, y otras variantes usadas), también se accede al PT. No solo hacer publicidad activa es importante, lo es también que haya indicaciones claras en SWA sobre dónde encontrar esa información.

Otro de los problemas significativos es el de la duplicidad, que afecta al 11\% de municipios de la muestra. Muchos casos encontrados se alejan de la garantía eficaz de transparencia, ya que responden, mayoritariamente, a esta tipología: i) ambos vacíos y ii) información complementaria por tipos de información recogida o por períodos (desde un año en uno, antes de ese año el otro), haciendo necesaria la consulta a ambos para acceder a toda la publicidad activa del municipio. Es importante que la información de transparencia se encuentre en una sola ubicación, y que se deshabilite uno de los PT en estos casos.

La vinculación del PT con la administración electrónica se impone con un $71,64 \%$ de PT en SE, lo que resulta entendible dada la obligatoriedad de la administración electrónica a partir del año 2020. Sin embargo, este hecho es el que parece haber provocado la duplicidad de PT de muchos municipios, puesto que la sede electrónica estatal (SEE) es la que más aparece en los casos de duplicidad. Existen casos de municipios que, teniendo otro PT, han deshabilitado el de SE estatal. Sin embargo, no ocurre así en la mayoría de los casos: el portal sigue activo y es, además, el único PT enlazado desde SWA a través de la SE del mismo. También son muchos los municipios que, teniendo solo el PT de SE estatal, lo tienen vacío. Este hecho deja claro, a nuestro juicio, el orden jerárquico que tiene la administración electrónica frente a la transparencia, quizá porque la primera conlleva sanciones concretas, y la segunda, no.

Así, a nuestro juicio, cualquier estudio futuro que aborde la evaluación de la transparencia municipal española, en especial de los tramos de menor población menos estudiados hasta ahora, debería tener en cuenta la diversidad y las posibles deficiencias de su realidad, que quedan patentes en este estudio. Dado que este trabajo gira en torno a la variable del tramo poblacional, las líneas de investigación futuras que estamos desarrollando ya abordarán tanto la perspectiva territorial como la de las metodologías usadas por los ayuntamientos para organizar y presentar su información. La finalidad de estas dos nuevas perspectivas será localizar las iniciativas que, desde ambas perspectivas, están resultando más exitosas en la práctica y, en la medida de lo posible, cuáles son las claves principales de su éxito. 
Por último, el cierre de este estudio se ha llevado a cabo en tiempo de la Covid-19. Se ha observado que la inmensa mayoría de las SWA tenían recogida información actualizada sobre las ayudas, indicaciones de desconfinamiento, etc., independientemente del tramo. Es decir, se puede ofrecer información actualizada y al día, cualquiera que sea el tamaño del municipio.

\section{Recomendaciones}

La existencia de SWA se presenta imprescindible para acceder tanto a la transparencia como a la administración electrónica; y de igual manera, se precisa la visibilidad de los enlaces a ambas, que en muchos casos cuesta localizar en la SWA. Es importante que las distintas administraciones, municipios y restantes administraciones, hagan el esfuerzo de que todos los municipios tengan SWA, tanto para el acceso a la información de transparencia como a la administración electrónica y que ambas se encuentren enlazadas desde lugares muy visibles y diferenciados.

Por otra parte, es obvio que no es suficiente con la presencia del PT si está vacío, o no se actualiza. Se ha observado en algunas CCAA, como Cataluña o Aragón, que ponen información relativa a composición política de los cargos municipales, plantilla, remuneraciones o presupuestos, entre otras, en los municipios de menor tramo que usan su PT. Es una iniciativa que puede ayudar a estos municipios que cuentan, por regla general, con menores recursos humanos y materiales.

En el caso de los municipios con duplicidad, sería conveniente que las administraciones inmediatamente superiores detectaran estos casos en sus territorios, y ayudasen a los municipios a eliminar uno de ellos, y concentrar en uno solo.

Por último, habría que hacer un esfuerzo por unificar los modelos por los que se organiza, presenta y evalúa la información en los PT. En este estudio se han detectado: ITA (de distintos años), LTBG, Leyes autonómicas, LTBG+Leyes autonómicas, Infoparticipa (con distinto número de items) y Dynamics Transparency Index (Dyntra); en este último, aunque no se haya usado en los estudios hasta la fecha, si ha aparecido en el análisis de la muestra. En algunos casos, dentro de la misma provincia e incluso el mismo tramo poblacional, se usan distintas organizaciones para acreditar la transparencia, lo que dificulta su seguimiento y evaluación, incluso a nivel provincial del mismo tramo. Si no es posible a nivel autonómico, al menos a nivel provincial se debería hacer un esfuerzo por consensuar los criterios de presentación y organización de la información para todos los municipios de los tramos poblacionales más pequeños.

\section{Notas}

1. El texto del acuerdo marco no se encuentra en ninguna sede web oficial actualmente. No obstante, se puede localizar en una búsqueda por el título en Internet. Además, se puede encontrar mención al mismo, y el enlace al listado de entidades locales que están usando ese portal de transparencia local, en la sebe web de la Federación Española de Municipios y Provincias (FEMP).

https://tinyurl.com/saboh9r

2. "En el marco de las reformas estructurales iniciadas por el Gobierno de la Nación, el pasado 26 de octubre de 2012 se acordó por el Consejo de Ministros la creación de una Comisión para la Reforma de las Administraciones Públicas (CORA) [...]

Entre otras medidas de reforma, CORA ha propuesto al Gobierno el establecimiento del Punto de Acceso General (PAG) como punto de entrada general, vía Internet, del ciudadano a las Administraciones Públicas. El fundamento de esta medida es la constatación de que en el momento actual existe una gran dispersión de la información de las Administraciones en distintos portales y webs, que provoca dificultades en el acceso de los ciudadanos a los procedimientos y servicios administrativos, informaciones duplicadas y falta de una coordinación adecuada en todas estas materias.

$[\ldots]$

Por su parte, el Real decreto 1671/2009, de 6 de noviembre, por el que se desarrolla parcialmente la Ley 11/2007, de 22 de junio, tanto en su preámbulo, como en sus artículos 7, 8, 9, 24 y 31, define las características básicas que deberá tener el Punto y señala que habrá de contener la sede electrónica que, en este ámbito, facilita el acceso a los servicios, procedimientos e informaciones accesibles de la Administración General del Estado y de los organismos públicos vinculados o dependientes de la misma.

$[\ldots]$

La citada ley 11/2007, de 22 junio, estableció el concepto de sede electrónica, que define en el artículo 10, apartado 1, como la dirección electrónica disponible para los ciudadanos a través de redes de telecomunicaciones cuya titularidad, gestión y administración corresponde a una Administración Pública, órgano o entidad administrativa en el ejercicio de sus competencias, señalando en el número 3 del mismo precepto que cada Administración Pública determinará las condiciones e instrumentos de creación de sus sedes electrónicas".

3. Existen más estudios sobre transparencia en el ámbito local español y de la administración electrónica centrados en algún aspecto de la transparencia: principalmente; el económico. En este artículo solo se han recogido los que se han realizado sobre transparencia en general y no solo sobre un aspecto, y que han sido publicados con posterioridad, o en el mismo año, a la aprobación de la LTBG. 
4. Aunque en el resumen se afirma haber hecho el estudio en ayuntamientos de más de 7.000 habitantes, en el texto sólo se ofrecen los datos de los de más de 10.000 .

5. Aunque el estudio de Pagán-Martínez et al. (2018) se centra en el acceso y visualización de la información en las webs de los municipios analizados, recoge también los items relativos a transparencia.

\section{Bibliografía}

Aguilera-Povedano, Manuel-Alejandro; Matas-Pastor, Joan-Josep; Durán-Mañes, Ángeles (2015). “Informe sobre la información publicada en las webs corporativas de los municipios de las Islas Baleares: resultados de la evaluación realizada de enero a abril de 2015". Informes Infoparticipa.

https://tinyurl.com/r2w3rel

Alonso-Magdaleno, María-Lorena; García-García, Jesús (2014). “Evaluación de la transparencia municipal en el Principado de Asturias". Auditoría pública, n. 64, pp. 75-86.

https://asocex.es/wp-content/uploads/PDF/Pag\%2075-86\%20\%20n\%C2\%BA\%2064.pdf

Cabezuelo-Lorenzo, Francisco; Rey-García, Pablo; Tapia-Frade, Alejandro (2016): "Análisis de las herramientas de control ciudadano sobre los representantes públicos: La transparencia informativa municipal en Castilla y León". Revista latina de comunicación social, n. 71, pp. 261-279.

https://doi.org/10.4185/RLCS-2016-1145

Comisionado de Transparencia de Canarias (2015). Informe anual de evaluación del cumplimiento de la Ley 12/2014 de transparencia de Canarias 2015. Edición del Comisionado de Transparencia y Acceso a la Información Pública de Canarias, $404 \mathrm{pp}$.

https://tinyurl.com/rzr3ecp

Comisionado de Transparencia de Canarias (2016). Informe anual de evaluación del cumplimiento de la Ley 12/2014 de Transparencia de Canarias 2016. Edición del Comisionado de Transparencia y Acceso a la Información Pública de Canarias, $377 \mathrm{pp}$.

https://tinyurl.com/vjx4ayt

Comisionado de Transparencia de Canarias (2017). Informe anual de evaluación del cumplimiento de la Ley 12/2014 de Transparencia de Canarias 2017. Edición del Comisionado de Transparencia y Acceso a la Información Pública de Canarias, $322 \mathrm{pp}$.

https://tinyurl.com/u2o3/95

Comisionado de Transparencia de Canarias (2018). Informe anual de evaluación del cumplimiento de la Ley 12/2014 de Transparencia de Canarias 2018. Evolución del Índice de Transparencia de Canarias ITCanarias 2016-2018.

https://tinyurl.com/ukyobx8

Delgado-Jalón, María-Luisa; Navarro-Heras, Emilio; Mora-Agudo, Leonor (2017). "Cumplimiento de los requisitos de transparencia: un diagnóstico de la situación para los municipios españoles de más de 50.000 habitantes". Innovar, v. 27, n. 66, pp. 109-121.

https://doi.org/10.15446/innovar.v27n66.66806

Delgado-Morales, Francisco (2015). "Sistemas de medición y transparencia en los ayuntamientos". Revista española de la transparencia, n. 0, pp. 12-20.

https://tinyurl.com/y8sgkz33

De-Vicente-Domínguez, Aída-María; Vera-Balanza, María-Teresa (2015). “Las webs de los ayuntamientos de Andalucía de municipios de más de 50.000 habitantes". En: Molina-Rodríguez-Navas, Pedro (coord.). Transparencia de la comunicación pública local. El mapa infoparticipa (www.mapainfoparticipa.com). Cuadernos artesanos de comunicación, n. 78. La Laguna (Tenerife): Sociedad Latina de Comunicación Social, pp. 49-67. ISBN: 9788415698951

https://doi.org/10.4185/cac78

España (2013). "Ley 19/2013, de 9 de diciembre, de transparencia, acceso a la información pública y buen gobierno". $B O E$, n. 295, 10 de diciembre [Texto consolidado: 6 de diciembre de 2018].

https://tinyurl.com/wt446wj

España (2014). "Orden HAP/1949/2014, de 13 de octubre, por la que se regula el Punto de Acceso General de la Administración General del Estado y se crea su sede electrónica". BOE, n. 260, 27 de octubre [Texto consolidado: 26 de noviembre de 2015].

https://tinyurl.com/tcdamhn

FEMP (2014). Ordenanza tipo de transparencia, acceso a la información y reutilización, aprobada en Junta de Gobierno FEMP de 27-5-2014.

https://tinyurl.com/se4qmww 
Fernández-Falero, María-Rosario; Trabadela-Robles, Javier; Garcés-Botacio, Indhira; Ruano-López, Soledad (2017). "Comunicación política de los ayuntamientos a través de sus webs. Caso de Extremadura". El profesional de la información, v. 26, n. 3, pp. 404-410.

https://doi.org/10.3145/epi.2017.may.06

Garrido-Rodríguez, Juan-Carlos; Zafra-Gómez, José-Luis (2017). “Evaluación del nivel de transparencia municipal. Aplicación al caso andaluz". Auditoría pública, n. 69, pp. 85-94.

https://asocex.es/evaluacion-del-nivel-de-transparencia-municipal-aplicacion-al-caso-andaluz

Guichot, Emilio (2015). “Entrada en vigor de la Ley de Transparencia en las entidades locales”. El blog de esPúblico, 9 de diciembre.

https://tinyurl.com/ww2k3fm

Herrero-Gutiérrez, Francisco-Javier; Martínez-Vallvey, Fernando; Tapia-Frade, Alejandro; Rey-García, Pablo; Cabezuelo-Lorenzo, Francisco (2017). "Transparencia en el sector público a través de salas de prensa online. Gestión de recursos colectivos y suinformación en webs municipales". El profesional de la información, v. 26, n. 3, pp. 421-429.

https://doi.org/10.3145/epi.2017.may.08

Herrero-Gutiérrez, Francisco-Javier; Ruano-López, Soledad (2017). “Transparencia municipal y participación ciudadana en la democracia española. Estudio de caso: Comunidad de Madrid". Chasqui. Revista latinoamericana de comunicación, n. 139, pp. 449-468.

https://tinyurl.com/y7agczr6

Manfredi-Sánchez, Juan-Luis; López-Cepeda, Ana-María; Álvarez-Peralta, Miguel; Saiz-Echezarreta, Vanesa; Reig-Cruañes, José (2016). "Transparencia y rendición de cuentas de los municipios de Castilla-La Mancha". Barataria. Revista castellano-manchega de ciencias sociales, n. 21, pp. 175-189.

http://dx.doi.org/10.20932/barataria.v0i21.304

Martínez-Roldán, Xabier; Piñeiro-Otero, Teresa; Baamonde-Silva, Xosé-Manuel (2016). “El desafío de la transparencia en la comunicación digital. Un estudio de caso sobre las webs municipales de Galicia y Norte de Portugal". Observatorio $\left(O B S^{*}\right)$, v. 10 , n. 4 , pp. 35-55.

https://tinyurl.com/rny87ry

Minhap-Femp (2015). Acuerdo marco de colaboración entre la Administración General del Estado (Ministerio de Hacienda y Administraciones Públicas [actual Ministerio de Política Territorial y Función Pública]) y la Federación Española de Municipios y Provincias (FEMP) para promover y facilitar el desarrollo de la Ley 19/2013, de 9 de diciembre, de transparencia, acceso a la información pública y buen gobierno en las entidades locales. Firmado el 28 de abril de 2015. https://bit.ly/3ppHAcf

Moreno-Sardá, Amparo; Corcoy-Rius, Marta; Molina-Rodríguez-Navas, Pedro; Toledano-Buendía, Samuel; Vera-Balanza, María-Teresa; Rodríguez-Breijo, Vanessa; Simelio-Solà, Núria (2015). "Comparativo de 230 webs de ayuntamientos de municipios de más de 20.000 habitantes". En: Molina-Rodríguez-Navas, Pedro (coord.). Transparencia de la comunicación pública local. El mapa infoparticipa (www.mapainfoparticipa.com). Cuadernos artesanos de comunicación, 78. La Laguna (Tenerife): Sociedad Latina de Comunicación Social, pp. 149-157. ISBN: 9788415698951

https://doi.org/10.4185/cac78

Moreno-Sardá, Amparo; Molina-Rodríguez-Navas, Pedro; Corcoy-Rius, Marta (2013). "La información de las administraciones públicas locales. Las webs de los ayuntamientos de Cataluña”. Revista latina de comunicación social, n. 68, pp. 502-528.

https://doi.org/10.4185/RLCS-2013-987

PAe (2019). Convenios en materia de Administración electrónica. Selección de convenios firmados con CCAA y otros organismos públicos. Portal de la Administración Electrónica. Gobierno de España.

https://tinyurl.com/ybyek5gw

Pagán-Martínez, María; Rodríguez-Muñoz, José-Vicente; Gonçalves-Sant'Ana, Ricardo-César; Lourenzani, Wagner-Luiz (2018). "Transparencia y acceso a la información electrónica en sedes electrónicas municipales". Informação \& sociedade, v. 28, n. 2, pp. 179-196.

https://periodicos.ufpb.br/ojs2/index.php/ies/article/view/31126

Paricio-Esteban, Pilar; Bruno-Carlos, Teresa; Alonso-Romero, Elvira; García-Alcober, María P. (2020). “Webs y portales de transparencia para la participación ciudadana en la gestión de las relaciones públicas con los stakeholders locales". Profesional de la información, v. 29, n. 3, e290335.

https://doi.org/10.3145/epi.2020.may.35 
Prieto-Sánchez, Cristina (2015). "Las webs de los ayuntamientos de la provincia de Granada”. En: Molina-Rodríguez-Navas, Pedro (coord.). Transparencia de la comunicación pública local. El mapa infoparticipa (www.mapainfoparticipa. com). Cuadernos artesanos de comunicación, n. 78. La Laguna (Tenerife): Sociedad Latina de Comunicación Social, pp. 87-98. ISBN: 9788415698951

https://doi.org/10.4185/cac78

Rebolledo, Marta; Zamora-Medina, Rocío; Rodríguez-Virgili, Jordi (2017). "Transparency in citizen participation tools and public information: A comparative study of the Spanish city councils' websites". El profesional de la información, v. 26, n. 3, pp. 361-369.

https://doi.org/10.3145/epi.2017.may.02

Ros-Medina, José-Luis (2018). “La evaluación de la transparencia en España a debate metodológico: mesta e índices de transparencia internacional". Revista internacional de transparencia e integridad, n. 6, pp. 1-22.

https://tinyurl.com/u5pv35r

Subires-Mancera, María-Purificación; Cuartero-Naranjo, Antonio (2015). "Las webs de los ayuntamientos de Andalucía de municipios de 10.001 a 50.000 habitantes". En: Molina-Rodríguez-Navas, Pedro (coord.). Transparencia de la comunicación pública local. El mapa infoparticipa (www.mapainfoparticipa.com). Cuadernos Artesanos de Comunicación, n. 78. La Laguna (Tenerife): Sociedad Latina de Comunicación Social, pp. 69-85. ISBN: 9788415698951 https://doi.org/10.4185/cac78

Trenta, Milena; Carrascosa-Puertas, Lara (2015). “Las webs de los ayuntamientos de Canarias de más de 50.000 habitantes". En: Molina-Rodríguez-Navas, Pedro (coord.). Transparencia de la comunicación pública local. El mapa infoparticipa (www.mapainfoparticipa.com). Cuadernos Artesanos de Comunicación, 78. La Laguna (Tenerife): Sociedad Latina de Comunicación Social, pp. 133-143. ISBN: 9788415698951

https://doi.org/10.4185/cac78

Vadillo-Bengoa, Nerea; Rodríguez-Breijo, Vanessa; Álvarez-Nobell, Alejandro (2015). "Las webs de los ayuntamientos de Aragón". En: Molina-Rodríguez-Navas, Pedro (coord.). Transparencia de la comunicación pública local. El mapa infoparticipa (www.mapainfoparticipa.com). Cuadernos artesanos de comunicación, n. 78. La Laguna (Tenerife): Sociedad Latina de Comunicación Social, pp. 113-131. ISBN: 9788415698951

https://doi.org/10.4185/cac78

Vera-Balanza, María-Teresa; López-Franco, Álvaro (2015). “Las webs de los ayuntamientos de la provincia de Cádiz”. En: Molina-Rodríguez-Navas, Pedro (coord.). Transparencia de la comunicación pública local. El mapa infoparticipa (www. mapainfoparticipa.com). Cuadernos artesanos de comunicación, n. 78. La Laguna (Tenerife): Sociedad Latina de Comunicación Social, pp. 99-111. ISBN: 9788415698951

https://doi.org/10.4185/cac78

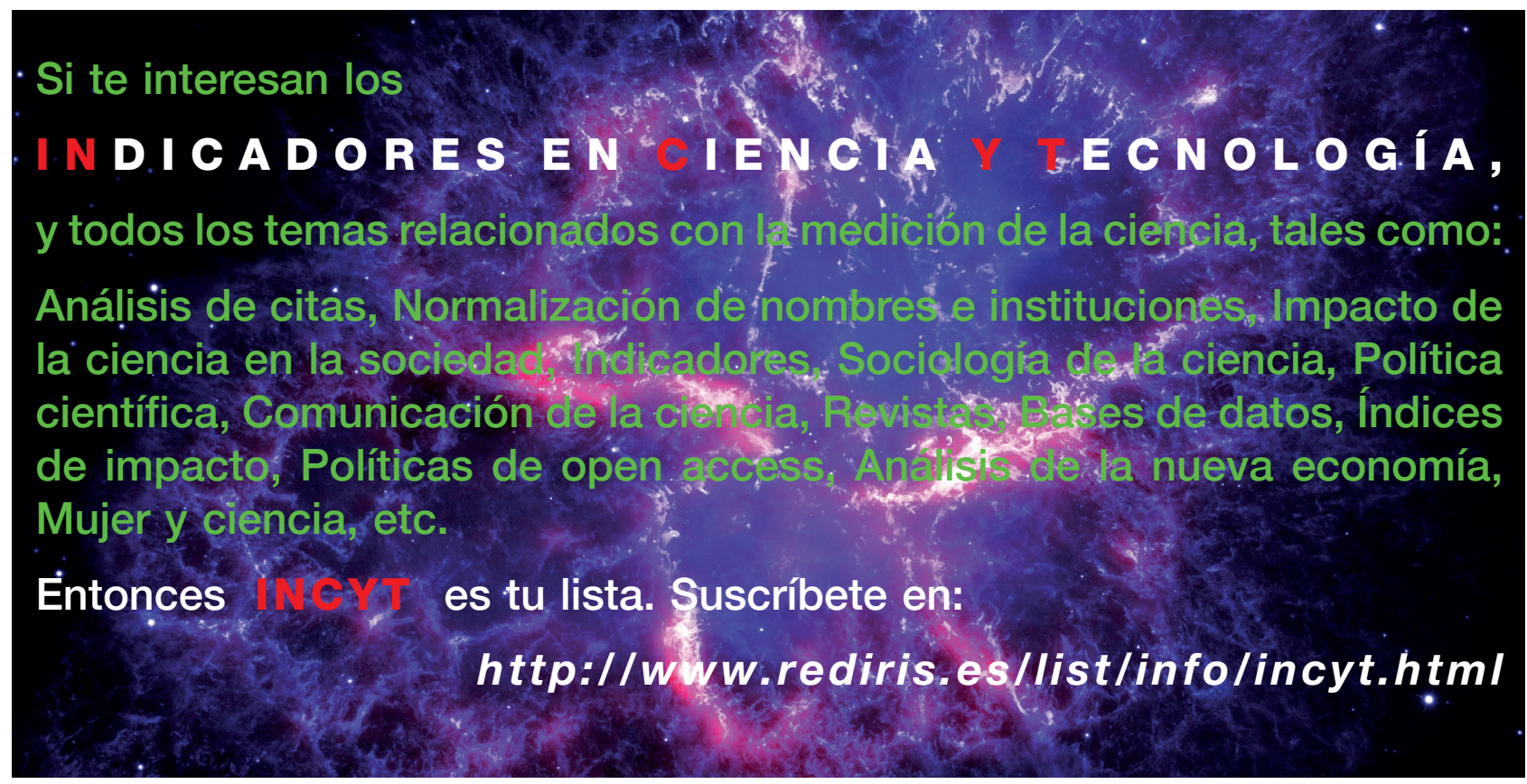

